\title{
Middle of the breasts pigmentation in a pedigree with POFUT1-related Dowling-Degos disease, expansion of the phenotype
}

\author{
Yingda Wu, Wanlu Zhang, Jianbing Wu, Yiping Ma, Pangen Cui, Chengrang Li
}

Department of Dermatology, Institute of Dermatology, Chinese Academy of Medical Sciences and Peking Union Medical College, Nanjing, Jiangsu, China

Adv Dermatol Allergol 2021; XXXVIII (3): 526-527 DOI: https://doi.org/10.5114/ada.2021.107939

Dowling-Degos disease (DDD [MIM: 179850, 615327 and 615674]), also called reticulate pigmented anomaly of the flexures (RPAF), is characterized by post-pubertal reticulate hyperpigmentation, particularly affecting the flexural areas and other great skin folds [1, 2]. As a rare autosomal dominant disorder, DDD-related genes include KRT5, POFUT1 and POGLUT1 genes [1-3].

We report a 37-year-old woman characterized by reticulated brown-black pigmented macules on the anterior chest, inframammary folds, dorsa of the neck, axillary folds, inguinal regions, flexor surfaces of the both wrist and inner sides of the thighs of 17 years' duration. All other systemic examinations were within normal limits. The parents of the proband were not consanguineous marriage. The family study unveiled that her paternal grandfather (I:1), father (II:1) and two aunts (II:3, II:4) suffer from a similar condition (Figure $1 \mathrm{~A}$ ).

The proband's DDD-related genes (KRT5, POFUT1 and POGLUT1) coding sequence and exon-intron junction analysis by Sanger sequencing revealed a heterozygous nonsense codon mutation c. 945T>G (p. Y315X) variant in exon 6 of the POFUT1 (Figure 1 B). This mutation was not detected in unaffected family members and 100 normal individuals (Figure $1 \mathrm{C}$ ). The c. 945T $>\mathrm{G}$ mutation causes the premature termination of O-fucosyltransferase-1's polymerization. This variant was not present in the $\mathrm{db}$ SNP or the HGMD.

Specifically, the proband presented with severe neck lesions and featured scattered comedones and pitted acneiform scars on the neck (Figure 1 D), which may hint the corresponding with acne inversa. A mixture of hyperpigmented and hypopigmented macules in the inner sides of wrists resembles features of dyschromatosis symmetrica hereditaria (Figure $1 \mathrm{E})$. Notably, this report is the first one to find the middle of the breasts as a clinical feature of DDD (Figure $1 \mathrm{~F}$ ). Though the middle of the breasts means a reticulate area, it has not been found before yet. In addition, inframammary folds involved in this report have been found in four already published articles.

The POFUT1 gene, as a member of the glycosyltransferase O-Fuc family, encodes the protein O-fucosyltransferase-1, which is composed by 388 amino acid residues with seven exons. The protein O-fucosyltransferase-1 supplies O-fucose to EGF (epidermal growth factor-like), prompting O-fucosylation of the Notch receptor signalling protein, which requires GDP-fucose as a substrate [4]. There are totally 16 GDP-Fucose binding sites on a conserved domain of O-FucT-1 varying from 30 to 379 residues. This novel nonsense mutation c. 945T>G (p. Y315X) is located in the conserved domain instead of a biting site, ending up with the coding of just 11 binding sites and generating a truncated protein. This truncated protein is largely non-functional and this consequence is supported by online software analysis.

Recently, PSENEN mutation carriers have been partly found to be caught in DDD and the inner cause of coexistence between DDD and acne inversa remains a mystery [5]. DDD is hypothesized to increase the susceptibility of acne inversa when exposed to some risk factors [5]. Interestingly, DDD and acne inversa were deemed the different subphenotypes of PSENEN mutation, when the hyperpigmentation is the main feature, we view it DDD; whereas when the follicle keratosis occupies the main location, we consider it acne inversa [6]. Further pathogenesis studies are needed to understand the development of DDD and other related skin diseases.

\footnotetext{
Address for correspondence: Pangen Cui, Chengrang Li, Department of Dermatology, Institute of Dermatology, Chinese Academy of Medical Sciences and Peking Union Medical College, 12 Jiang Wang Miao St, Xuanwu District, Nanjing, 210042, China, e-mail: cuipangen@126.com, dr_lcr72@163.com Received: 8.09 .2019 , accepted: 6.10 .2019$.
} 

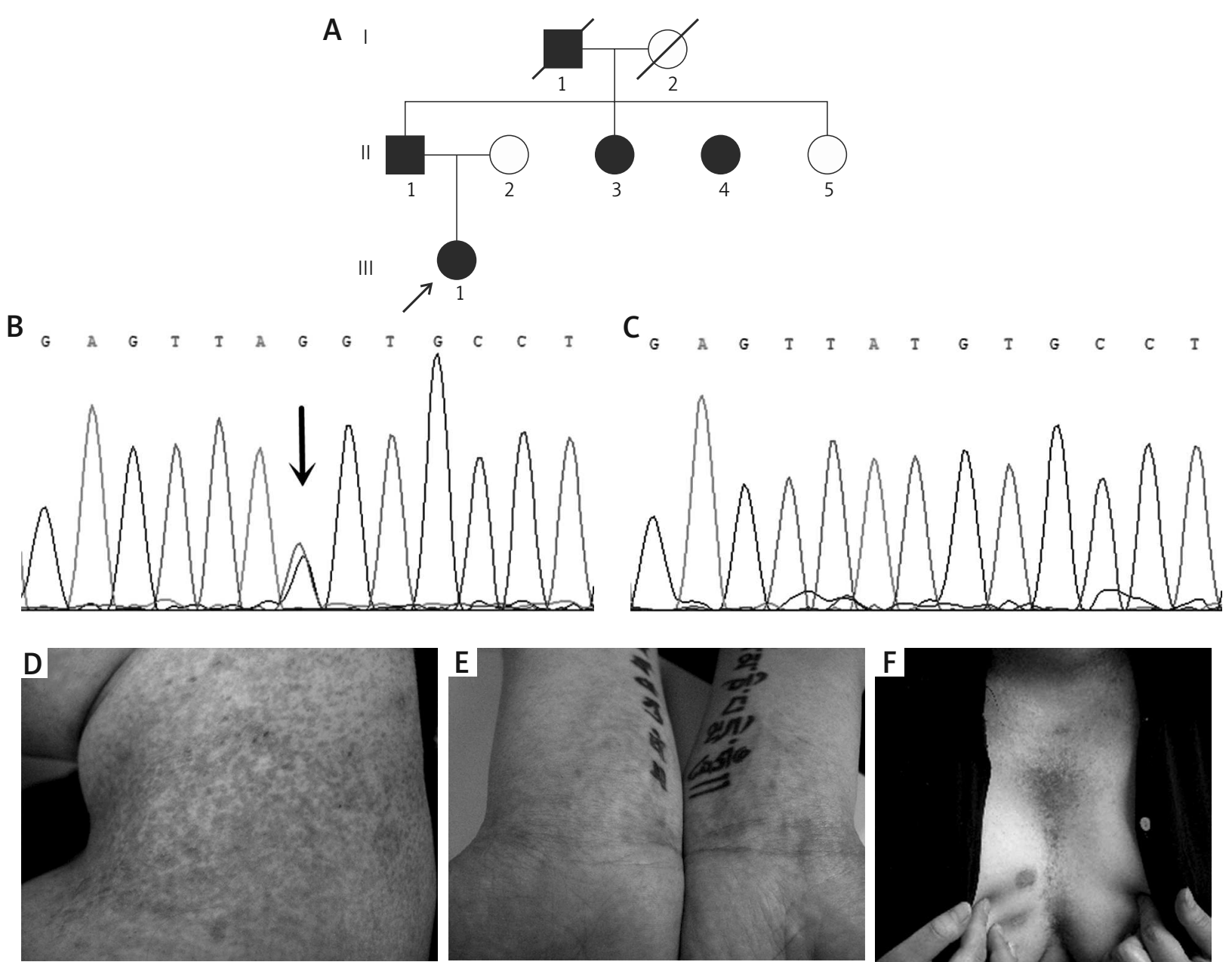

Figure 1. POFUT1 mutations and clinical appearance of the patients. A - Genealogical tree of the proband's family. B - The gene mutation result: c. 945T>G (p. Y315fs). C - Wild-type sequence of the gene mutation. D - Proband's neck lesions. $\mathrm{E}$ - Proband's inner sides of wrists presentation. $\mathbf{F}$ - Proband's chest lesions

In conclusion, we report a DDD family with a novel gene mutation and we are first to find a new clinical feature involving areas in the middle of breasts, which enrich the phenotype, gene mutation database and contribute to genetic counselling to the patients' family.

\section{Acknowledgments}

This work was supported by the CAMS Innovation Fund for Medical Sciences (2016-I2M-1-002), Project of Six Talent Peaks (WSN-126).

\section{Conflict of interest}

The authors declare no conflict of interest.

\section{References}

1. Li M, Cheng R, Liang J, et al. Mutations in POFUT1, encoding protein O-fucosyltransferase 1, cause generalized DowlingDegos disease. Am J Hum Genet 2013; 92: 895-903.
2. Betz RC, Planko L, Eigelshoven S, et al. Loss-of-function mutations in the keratin 5 gene lead to Dowling-Degos disease. Am J Hum Genet 2006; 78: 510-9.

3. Basmanav FB, Oprisoreanu A, Pasternack SM, et al. Mutations in POGLUT1, encoding protein o-glucosyltransferase 1, cause autosomal-dominant Dowling-Degos disease. Am J Hum Genet 2014; 94: 135-43.

4. Zhang J, Li M, Yao Z. Updated review of genetic reticulate pigmentary disorders. B J Dermatol 2017; 177: 945-59.

5. Ralser DJ, Basmanav FBÜ, Tafazzoli A, et al. Mutations in $\gamma$-secretase subunit-encoding PSENEN underlie DowlingDegos disease associated with acne inversa. J Clin Invest 2017; 127: 1485-90.

6. Li C, Li W, Xu H, et al. PSENEN mutation carriers with comanifestation of acne inversa (Al) and Dowling-Degos disease (DDD): is Al or DDD the subphenotype? I Invest Dermatol 2017: 137: 2234-6. 